ANNALES

UNIVERSITATIS MARIAE CURIE-SKŁODOWSKA

L UBLIN - POLONIA

VOL. LXV, NO. 1, 2011

SECTIO A

$49-67$

AHMAD RAMI EL-NABULSI

\title{
Extended fractional calculus of variations, complexified geodesics and Wong's fractional equations on complex plane and on Lie algebroids
}

\begin{abstract}
In this work, we communicate the topic of complex Lie algebroids based on the extended fractional calculus of variations in the complex plane. The complexified Euler-Lagrange geodesics and Wong's fractional equations are derived. Many interesting consequences are explored.
\end{abstract}

1. Introduction. The concept of fractional derivative and integral dates from the origin of calculus itself. It was founded in fact by Liouville between 1832 and 1837. However, in 1876, Riemann introduced the fractional integral and accordingly, the definition of Riemann-Liouville (RL) fractional integro-differentiation was recognized and has become most widely used. Nevertheless, when dealing with a dynamical system, we are unable to apply non-zero initial conditions with real life measurable quantities making use of the RL fractional integral. This was done only in 1967 by Caputo, who he introduced the well-known Caputo fractional derivative in a way similar to RL, but only with a change of the order of integration and differentiation. Caputo fractional derivative has become lately well-liked in the area of algorithm development. The reader is referred to Podlubny book for a summary of basic techniques, geometrical and physical interpretation

2000 Mathematics Subject Classification. 49S05, 26A33, 20 L05.

Key words and phrases. Extended fractional calculus, complex plane, complex Lie algebroids. 
of fractional derivatives and integrals [57]. It is noteworthy that fractional calculus plays a crucial role in dynamical systems governed by fractional differential equations as the memory effect of the convolution of the fractional integral gives the equation amplified significant power [54, 59, 53]. This amazing fact was proved to play a crucial role in different branches of sciences and engineering, including viscoelasticity, viscoplasticity, damping and diffusion $[42,60,55,3,56,61,62]$. Furthermore, fractional derivatives have memory effect and hence one naturally expects interesting consequences in modeling behaviour of complex classical and quantum dynamical systems $[36,35,37,40,41]$.

On the other hand, in recent years, plentiful works have been dedicated to one important branch of fractional calculus which is the "Fractional Calculus of Variations" (FCV). We believe today after a series of papers that the FCV play a leading role in nonconservative dynamical system where traditional Lagrangian and Hamiltonian mechanics based on the standard calculus of variations do not hold and fail to describe correctly the dissipative behavior $[58,1,2,5,6,25,24]$. Nevertheless, the largest part of the approaches discussed in literature deal with Riemann-Liouville derivatives and integrals, e.g. the Fractional ActionLike Variational Approach (FALVA) $[9,10]$ and a few with Caputo or Riesz derivatives and integrals [31]. Depending on the category of functional integral considered, different fractional Euler-Lagrange type equations were obtained. Necessary optimality conditions of Euler-Lagrange type for the fundamental fractional problem of the calculus of variations were obtained and discussed under appropriate convexity assumptions $[28,27,29,30,32,4]$. However, we believe that the concept of FCV could be introduced in a number of ways making use of different types of fractional operators, e.g. Erdélyi-Kober integral, Saxena-Kumbhat hypergeometric integral, generalized single-time Stieltjes fractal-fractional integral, extended exponentially fractional integral and so on $[12,23,20,21]$. These new concepts were proved to play a critical role in open one-dimensional dynamical systems with only one degree of freedom exhibiting an exponentially increasing or decaying mass and varying periodical frequency, e.g. quantum Bateman-Feshbach-Tikochinsky and Caldirola-Kanai damped harmonic oscillators [16, 17].

Further extended scenario of FCV was introduced more recently by the author of the present paper and entitled "periodic functional approach" based on a periodic fractional kernel inside the integral action [21]. The new approach was proved to play a vital role in modern cosmology. In this work, we introduce a new kind of extended exponentially FCV with complex fractional derivatives. Our main aim is to derive the fractional Euler-Lagrange equation on complex Lie algebroids, i.e. complex plane. The notion of Lie algebroid is in reality a generalization of both the concept of Lie algebra and the concept of an integrable distribution. The category 
of Lie algebroids has proved to be useful in the formulation and analysis of numerous problems in differential geometry.

Given that a Lie algebroid is a notion which merely unifies tangent bundles and Lie algebras, one expects their relation to Lagrangian and Hamiltonian classical mechanics. In the context of classical mechanics, Weinstein was the first who developed a theory of Lagrangian and Hamiltonian systems on Lie algebroids and their discrete analog on Lie groupoids [63, 64]. In the last years, Weinstein's theory has been developed by many authors, and accordingly, an influential mathematical structure was emerged and ranged from classical to generalized complex Lie algebroids with vakonomic and nonholonomic constraints. Weinstein's theory plays as well a crucial role in symmetry problems $[50,51,52]$. Symmetries are mathematically described by parameter groups of transformations. Their significance ranges from elementary and theoretical aspects to physical applications, having thoughtful implications in the dynamical behavior of the systems and in their basic qualitative properties. Nevertheless, we are also aware of the importance of symmetry issue in Lie algebroids throughout mathematics and of the fact that the manifolds of Lie groups and their homogeneous spaces provide accessible examples on which to do geometry and analysis. This is made promising by the fact that Lie groups may be studied in terms of their Lie algebras: the Lie algebra is a linear space and the entire linear algebra techniques may be brought to stand on it. This issue plays also a crucial role in Connes's noncommutative geometry $[7,8,46]$. It is noteworthy that Lie algebroids generalize Lie algebras, and in certain quantum systems they represent extended quantum (algebroid) symmetries.

However, a basic motivation for the study of fractional calculus of variations on complex Lie algebroids is a description of the renowned results for some moduli spaces of holomorphic structures on a complex vector bundle over a compact complex manifold in a unified handling, e.g. the related Hitchin-Kobayashi correspondence and the moduli space of Higgs bundles on a compact Riemann surface $[49,33,34,38,50,44]$. More recent examples of this phenomenon involve the moduli space of complex B-branes and the moduli space of symplectic A-branes, based on generalized complex geometry. They play an extremely important role in mirror symmetry and geometric Langland's program [47, 48]. These reasons motivate to find an extended fractional action-like variational description of Lagrange's equations for a Lagrangian system defined on a more general Lie algebroid.

The paper is organized as follows: in Section 2, we introduce the basic concepts of extended exponentially FCV with complex fractional derivative on complex plane. In Section 3, we introduce the Lagrangian concepts on complex Lie algebroids. The resulting complexified Euler-Lagrange, 
geodesics and Wong's equations are discussed in the same section. In Section 4, we initiate the complexified Hamiltonian formalism on complex Lie algebroids. Conclusions and perspectives are discussed in Section 5.

2. Extended complexified fractional action-like variational approach. We start by introducing the following definition:

Definition 2.1 (Extended Complexified Fractional Integral (ECFI)). Let $f$ be an analytic function in a simply connected region of the $z$-plane containing the origin. The ECFI of order $\alpha>0$, is defined by:

$$
K_{(z)}^{(-\alpha)} f(z)=\frac{1}{\Gamma(\alpha)} \int_{0}^{z} f(\zeta)(\cosh z-\cosh \zeta)^{\alpha-1} d \zeta
$$

where the multiplicity of $(\cosh z-\cosh \zeta)^{\alpha-1}$ is removed by requiring $\log (\cosh z-\cosh \zeta)$ to be real when $\cosh z-\cosh \zeta>0$. Here $\Gamma(\alpha)$ is the Euler gamma function [26].

Definition 2.2. Let $f$ be an analytic function in a simply connected region of the $z$-plane containing the origin. The Riesz-Caputo complex fractional derivative of order $0 \leq \alpha<1$, is defined by:

$$
D_{(z)}^{(\alpha)} f(z)=\frac{1}{\Gamma(1-\alpha)} \frac{d}{d z} \int_{0}^{z}(\cosh z-\cosh \zeta)^{-\alpha} f(\zeta) d \zeta,
$$

where the multiplicity of $(\cosh z-\cosh \zeta)^{\alpha-1}$ is removed by requiring $\log (\cosh z-\cosh \zeta)$ to be real when $\cosh z-\cosh \zeta>0$.

Definition 2.3. Given a smooth Lagrangian function in a simply connected region of the $z$-plane containing the origin assumed to be a $C^{2}$-function with respect to all its arguments. The extended complexified fractional action is defined by

$$
S_{(z)}^{(-\alpha)} f(z)=\frac{1}{\Gamma(\alpha)} \int_{0}^{z} L\left(\zeta, q(\zeta), D_{(z)}^{(\alpha)} q(\zeta)\right)(\cosh z-\cosh \zeta)^{\alpha-1} d \zeta
$$

under given boundary condition $q(0)=q_{0}$.

To define the corresponding extended fractional variational action and consequently the fractional variational problem on real or complex Lie algebroids, we have to identify two basic objects: an infinite-dimensional manifold $M$ of paths whose tangent space $T M$ represents all possible variations and an action functional on $M[50,51,52]$. Then, we have to choose a submanifold of admissible paths and a set of admissible variations of admissible paths.

In fact, given a vector bundle $\pi: E \rightarrow M$ given by a vector bundle map $\rho: E \rightarrow T M$ over the identity in $M$ called the anchor map, a smooth Lagrangian function $L: E \rightarrow \mathbb{R}$, together with a Lie algebra structure on the $C^{\infty}(M)$-module of sections of $E$ determined by the Lie bracket. In 
turn, the Lie bracket induces a Lie algebra homomorphism $\bar{\rho}$ of $C^{\infty}(M)$ from $\operatorname{Sec}(E) \rightarrow \chi(M)$ by the anchor map $\rho: E \rightarrow T M$ given by:

$$
\begin{gathered}
\sigma \in \operatorname{Sec}(E) \rightarrow \bar{\rho}(\sigma)(q) \in \chi(M) \\
\bar{\rho}(\sigma)(q)=\rho(s(q)), \forall q \in M
\end{gathered}
$$

satisfying the Leibniz compatibility identity:

$$
[\sigma, f \eta]_{E}=(\rho(\sigma) f) \eta+f[\sigma, \eta]_{E}, \forall f \in C^{\infty}(M), \sigma, \eta \in \operatorname{Sec}(E) .
$$

Definition 2.4. A vector bundle $(E, \xi, M)$ endowed with a Lie algebroid structure $\left([\cdot, \cdot]_{E}, \rho\right)$ is called Lie algebroid over $M$ and is denoted by the triple $\left(E,[\cdot, \cdot]_{E}, \rho\right)$.

A local coordinate system $\left(q^{i}\right)$ in the base manifold $M$, and a local basis of section $\left(e_{\gamma}\right)$ of $E$ determine a local coordinate system $\left(q^{i}, y^{\eta}\right)$ on $E$. The anchor and the bracket are locally determined by the structure functions

$$
\rho_{i}^{\gamma}, C_{i j}^{k} \in C^{\infty}(M) \text { of }\left(E,[\cdot, \cdot]_{E}, \rho\right)
$$

with

$$
\begin{gathered}
\bar{\rho}\left(e_{i}\right)=\rho_{i}^{\eta} \frac{\partial}{\partial q^{n}} \equiv \rho_{i}^{\eta} \partial_{\eta}, \\
\partial_{\eta} \doteq \frac{\partial}{\partial q^{n}}, \\
{\left[e_{i}, e_{j}\right]=C_{i j}^{k} e_{k},}
\end{gathered}
$$

which satisfies the following structure relations, which results from the Leibniz identity and the Jacobi identity:

$$
\begin{array}{r}
\rho_{i}^{\eta} \frac{\partial \rho_{j}^{\sigma}}{\partial q^{\eta}}-\rho_{j}^{\eta} \frac{\partial \rho_{i}^{\sigma}}{\partial q^{\eta}}=\rho_{k}^{\sigma} C_{i j}^{k}, \eta=\overline{1, N}, i=\overline{1, N}, \\
\sum_{\operatorname{cyclic}(i, j, k)}\left(\rho_{i}^{\eta} \frac{\partial C_{j k}^{l}}{\partial q^{\eta}}+C_{j k}^{h} C_{i h}^{l}\right)=0 .
\end{array}
$$

This innovative formalism was proved in recent years to be a powerful tool to investigate many fundamental properties of a given complex dynamical systems. Complex Lie algebroid structures on complex vector bundles over real bases can be defined similarly, replacing the tangent bundle of the base by the complexified tangent bundle. We consider at the moment the space of $E$-paths on the complex Lie algebroid denoted by $\mathcal{P}(J, E), J=[0, z]$, which is a differentiable Banach manifold.

Theorem 2.1 ([43]). Let the manifold $M$ be the space of all $C^{1}$-paths $[0, z] \rightarrow E$ in $E$ and $L: E \rightarrow \mathbb{C}$ be a smooth Lagrangian function in a simply connected region of the $z$-plane containing the origin assumed to be a $C^{2}$-function with respect to all its arguments on the complex Lie algebroid $E$ with admissible curve $q$ in $E$ and with two fixed endpoints

$$
A, B \in M \subset \mathcal{P}(J, E)_{A}^{B}=\{\mathcal{P}(J, E) \mid \pi(q(0))=A \text { and } \pi(q(z))=B\} .
$$


Let $\tau_{L}=\left(\partial L / \partial y^{i}\right) d y^{i}$ in local coordinate. The critical points of the complexified fractional action integral $I: \mathcal{P}(J, E) \rightarrow \mathbb{R}$ defined by the extended fractional complexified action (3) on the complex Banach manifold $\mathcal{P}(J, E)_{A}^{B}$ are exactly those elements of that space which satisfy the following extended complexified fractional Euler-Lagrange equation:

$$
\delta^{r} L\left(\zeta, q(\zeta), D_{(z)}^{(\alpha)} q(\zeta)\right)=0
$$

where

$$
\begin{aligned}
\left\langle\delta^{r} L\left(\zeta, q(\zeta), D_{(z)}^{(\alpha)} q(\zeta)\right)\right\rangle= & \left\langle d L\left(\zeta, q(\zeta), D_{(z)}^{(\alpha)} q(\zeta)\right), \sum_{q}(\sigma)\right\rangle \\
& -D_{(z)}^{(\alpha)}\left\langle z_{L\left(\zeta, q(\zeta), D_{(z)}^{(\alpha)} q(\zeta)\right)} \circ q, \sigma\right\rangle \\
& -\left[\frac{(\alpha-1) \sinh \zeta}{\cosh z-\cosh \zeta}\right]\left\langle z_{L\left(\zeta, q(\zeta), D_{(z)}^{(\alpha)} q(\zeta)\right)} \circ q, \sigma\right\rangle .
\end{aligned}
$$

Proof. Let $q \in \mathcal{P}(J, E)_{A}^{B}$, the tangent space on $\mathcal{P}(J, E)_{A}^{B}$ is the set of complex vector fields along $q$ of the form:

$$
\Xi_{q}(\sigma): \sigma \in \operatorname{Sec}(E) / \sigma(a)=\sigma(b)=0 .
$$

The action (3) is smooth, then

$$
\begin{aligned}
& 0=\left\langle d S_{(z)}^{(-\alpha)}, \Xi_{q}(f \sigma)\right\rangle \\
& =\frac{1}{\Gamma(\alpha)} \int_{0}^{z}\left\langle L\left(\zeta, q(\zeta), D_{(z)}^{(\alpha)} q(\zeta)\right), \Xi_{q}(f \sigma)\right\rangle(\cosh z-\cosh \zeta)^{\alpha-1} d \zeta \\
& =\frac{1}{\Gamma(\alpha)}\left(\int _ { 0 } ^ { z } \left[f(\zeta)\left\langle d L\left(\zeta, q(\zeta), D_{(z)}^{(\alpha)} q(\zeta)\right), \Xi_{q}(\sigma)\right\rangle\right.\right. \\
& -D_{(z)}^{(\alpha)} \frac{d}{d \zeta}\left\langle z_{L\left(\zeta, q(\zeta), D_{(z)}^{(\alpha)} q(\zeta)\right)} \circ q, \sigma\right\rangle(\cosh z-\cosh \zeta)^{\alpha-1} \\
& \left.+\frac{d}{d \zeta}\left[(\cosh z-\cosh \zeta)^{\alpha-1}\right]\left\langle\tau_{L\left(\zeta, q(\zeta), D_{(z)}^{(\alpha)} q(\zeta)\right)} \circ q, \sigma\right\rangle\right] d \zeta \\
& \left.+\left.f(\zeta)\left\langle\tau_{L\left(\zeta, q(\zeta), D_{(z)}^{(\alpha)} q(\zeta)\right)} \circ q, \sigma\right\rangle(\cosh z-\cosh \zeta)^{\alpha-1}\right|_{0} ^{z}\right) .
\end{aligned}
$$

Using the fact that

$$
\Xi_{q}(f(\zeta) \sigma(\zeta))=f(\zeta) \sum_{q}(\sigma(\zeta))+\frac{d f(\zeta)}{d \zeta} \sigma_{q}^{v}
$$

we obtain after simple algebraic manipulation

$$
\left\langle d S_{(z)}^{(-\alpha)}, \Xi_{q}(f(\zeta) \sigma(\zeta))\right\rangle=\int_{0}^{z} f(\zeta)\left\langle\delta^{r} L\left(\zeta, q(\zeta), D_{(z)}^{(\alpha)} q(\zeta)\right), \sigma(\zeta)\right\rangle d \zeta
$$


Here $v=\rho(q)$ is the actual velocity and $d \sigma_{q}^{v} / d \zeta$ is the Riemann-Liouville complex fractional derivative of the canonical vertical lift of $\sigma$. This equation is satisfied for every analytic function defined in a simply connected region of the $z$-plane containing the origin and for every section $\sigma \in \operatorname{Sec}(E)$. Thus, the critical points are satisfied by

$$
\delta^{r} L\left(\zeta, q(\zeta), D_{(z)}^{(\alpha)} q(\zeta)\right)=0
$$

Corollary 2.1. The fractional complex Euler-Lagrange equation in complex local coordinates is

$$
\begin{gathered}
\rho_{i}^{\eta} \frac{\partial L\left(\zeta, q(\zeta), D_{(z)}^{(\alpha)} q(\zeta)\right)}{\partial x^{\eta}}-D_{(z)}^{(\alpha)} \frac{\partial L\left(\zeta, q(\zeta), D_{(z)}^{(\alpha)} q(\zeta)\right)}{\partial y^{i}} \\
-\frac{\partial L\left(\zeta, q(\zeta), D_{(z)}^{(\alpha)} q(\zeta)\right)}{\partial y^{k}} C_{i j}^{k} y^{j} \\
-\frac{(\alpha-1) \sinh \zeta}{\cosh z-\cosh \zeta} \frac{\partial L\left(\zeta, q(\zeta), D_{(z)}^{(\alpha)} q(\zeta)\right)}{\partial y^{i}}=0,
\end{gathered}
$$

where

$$
\dot{x}^{\eta} \equiv \frac{d x^{\eta}}{d \zeta}=\rho_{i}^{\eta} y^{i}
$$

Remark 2.1. If for instance we set $\zeta^{\eta}=\zeta_{1}^{\eta}+i \zeta_{2}^{\eta}, x^{\eta}=x_{1}^{\eta}+i x_{2}^{\eta}$ and $y^{j}=y_{1}^{j}+i y_{2}^{j}$, then the complexified Lagrangian is split into a real and a complex part as follows: $L=L_{1}+i L_{2}$. The following rules are useful:

$$
\begin{gathered}
\frac{\partial}{\partial x^{\eta}}=\frac{1}{2}\left(\frac{\partial}{\partial x_{1}^{\eta}}-i \frac{\partial}{\partial x_{2}^{\eta}}\right), \\
\frac{\partial}{\partial y^{i}}=\frac{1}{2}\left(\frac{\partial}{\partial y_{1}^{i}}-i \frac{\partial}{\partial y_{2}^{i}}\right), \\
\rho_{i}^{\eta} \frac{\partial \rho_{j}^{\sigma}}{\partial q_{1}^{\eta}}-\rho_{j}^{\eta} \frac{\partial \rho_{i}^{\sigma}}{\partial q_{1}^{\eta}}-i\left(\rho_{i}^{\eta} \frac{\partial \rho_{j}^{\sigma}}{\partial q_{2}^{\eta}}-\rho_{j}^{\eta} \frac{\partial \rho_{i}^{\sigma}}{\partial q_{2}^{\eta}}\right) \equiv\left[\rho_{k}^{\sigma} C_{i j}^{k}\right]_{1}-i\left[\rho_{k}^{\sigma} C_{i j}^{k}\right]_{2} \\
\equiv \rho_{k}^{\sigma}\left(\Re\left[C_{i j}^{k}\right]_{1}-i \Im\left[C_{i j}^{k}\right]_{2}\right) \equiv \rho_{k}^{\sigma} \tilde{C}_{i j}^{k},
\end{gathered}
$$

where

$$
\tilde{C}_{i j}^{k}=\Re\left[C_{i j}^{k}\right]_{1}-i \Im\left[C_{i j}^{k}\right]_{2} \equiv \bar{C}_{i j}^{k}-i \underline{C}_{i j}^{k} .
$$

Remark 2.2. By letting $z=x+i y$ and $\zeta=\zeta_{1}+i \zeta_{2}$, we may split the Riesz-Caputo complex fractional derivative into a real and a complex part 
as follows:

$$
\begin{aligned}
& D_{(z)}^{(\alpha)} f(x)=\frac{1}{2 \Gamma(1-\alpha)}\left(\frac{d}{d x}-i \frac{d}{d y}\right) \\
& \quad \times \int_{0}^{x+i y}\left(\cosh (x+i y)-\cosh \left(\zeta_{1}+i \zeta_{2}\right)\right)^{-\alpha}\left(F\left(\zeta_{1}\right)+i G\left(\zeta_{2}\right)\right)\left(d \zeta_{1}+i d \zeta_{2}\right) \\
&=\frac{1}{2 \Gamma(1-\alpha)}\left(\frac{d}{d x}-i \frac{d}{d y}\right) \int_{0}^{x+i y}\left(\cosh (x+i y)-\cosh \left(\zeta_{1}+i \zeta_{2}\right)\right)^{-\alpha} \\
& \quad \times\left[\left(F\left(\zeta_{1}\right) d \zeta_{1}-G\left(\zeta_{2}\right) d \zeta_{2}\right)+i\left(F\left(\zeta_{1}\right) d \zeta_{2}+G\left(\zeta_{2}\right) d \zeta_{1}\right)\right]
\end{aligned}
$$

Making use of the simple decomposition

$$
\begin{aligned}
\cosh (x+i y) & -\cosh \left(\zeta_{1}+i \zeta_{2}\right) \\
& =\cos y \cosh x-\cos \zeta_{2} \cosh \zeta_{1}+i\left(\sin y \sinh x-\sin \zeta_{2} \sinh \zeta_{1}\right),
\end{aligned}
$$

and the rule [65]

$$
(X+i Y)^{-\alpha}=R^{-\alpha} \exp (-i Y \varphi \alpha)=R^{-\alpha}[\cos (\alpha Y \varphi)-i \sin (\alpha Y \varphi)],
$$

where

$$
\begin{gathered}
R=\sqrt{X^{2}+Y^{2}}, \quad \varphi=\frac{\pi}{2}-\arcsin \frac{X}{R}, \\
X=\cosh y \cosh x-\cos \zeta_{2} \cosh \zeta_{1}, \quad Y=\sin y \sinh x-\sin \zeta_{2} \sinh \zeta_{1},
\end{gathered}
$$

we obtain after simple algebraic manipulation

$$
\begin{aligned}
D_{(z)}^{(\alpha)} f(x)=\frac{R^{-\alpha}}{2 \Gamma(1-\alpha)}[ & \frac{d}{d x} \int_{0}^{x+i y}\left[\cos (\alpha Y \varphi)\left(F\left(\zeta_{1}\right) d \zeta_{1}-G\left(\zeta_{2}\right) d \zeta_{2}\right)\right. \\
& \left.+\sin (\alpha Y \varphi)\left(F\left(\zeta_{1}\right) d \zeta_{2}-G\left(\zeta_{2}\right) d \zeta_{1}\right)\right] \\
& +\frac{d}{d y} \int_{0}^{x+i y}\left[\cos (\alpha Y \varphi)\left(F\left(\zeta_{1}\right) d \zeta_{2}+G\left(\zeta_{2}\right) d \zeta_{1}\right)\right. \\
& \left.\left.-\sin (\alpha Y \varphi)\left(F\left(\zeta_{1}\right) d \zeta_{1}-G\left(\zeta_{2}\right) d \zeta_{2}\right)\right]\right] \\
+i \frac{R^{-\alpha}}{2 \Gamma(1-\alpha)}[ & \frac{d}{d x} \int_{0}^{x+i y}\left[\cos (\alpha Y \varphi)\left(F\left(\zeta_{1}\right) d \zeta_{2}+G\left(\zeta_{2}\right) d \zeta_{1}\right)\right. \\
& \left.-\sin (\alpha Y \varphi)\left(F\left(\zeta_{1}\right) d \zeta_{1}-G\left(\zeta_{2}\right) d \zeta_{2}\right)\right] \\
& -\frac{d}{d y} \int_{0}^{x+i y}\left[\cos (\alpha Y \varphi)\left(F\left(\zeta_{1}\right) d \zeta_{1}-G\left(\zeta_{2}\right) d \zeta_{2}\right)\right. \\
& \left.\left.+\sin (\alpha Y \varphi)\left(F\left(\zeta_{1}\right) d \zeta_{2}+G\left(\zeta_{2}\right) d \zeta_{1}\right)\right]\right]
\end{aligned}
$$


However,

$\frac{d}{d y} \int_{0}^{x+i y}\left[\cos (\alpha Y \varphi)\left(F\left(\zeta_{1}\right) d \zeta_{2}+G\left(\zeta_{2}\right) d \zeta_{1}\right)-\sin (\alpha Y \varphi)\left(F\left(\zeta_{1}\right) d \zeta_{1}-G\left(\zeta_{2}\right) d \zeta_{2}\right)\right]$,

and

$\frac{d}{d y} \int_{0}^{x+i y}\left[\cos (\alpha Y \varphi)\left(F\left(\zeta_{1}\right) d \zeta_{1}-G\left(\zeta_{2}\right) d \zeta_{2}\right)+\sin (\alpha Y \varphi)\left(F\left(\zeta_{1}\right) d \zeta_{2}+G\left(\zeta_{2}\right) d \zeta_{1}\right)\right]$,

will both acquire complex values and accordingly by denoting:

$$
\begin{gathered}
\frac{d}{d x} \int_{0}^{x+i y}\left[\operatorname { c o s } ( \alpha Y \varphi ) \left(F\left(\zeta_{1}\right) d \zeta_{1}\right.\right. \\
\left.\left.\quad-G\left(\zeta_{2}\right) d \zeta_{2}\right)+\sin (\alpha Y \varphi)\left(F\left(\zeta_{1}\right) d \zeta_{2}+G\left(\zeta_{2}\right) d \zeta_{1}\right)\right]=D_{1(x)}^{\alpha} f, \\
\frac{d}{d x} \int_{0}^{x+i y}\left[\cos (\alpha Y \varphi)\left(F\left(\zeta_{1}\right) d \zeta_{2}+G\left(\zeta_{2}\right) d \zeta_{1}\right)\right. \\
\left.\quad-\sin (\alpha Y \varphi)\left(F\left(\zeta_{1}\right) d \zeta_{1}-G\left(\zeta_{2}\right) d \zeta_{2}\right)\right]=D_{2(x)}^{\alpha} f, \\
\frac{d}{d y} \int_{0}^{x+i y}\left[\cos (\alpha Y \varphi)\left(F\left(\zeta_{1}\right) d \zeta_{2}+G\left(\zeta_{2}\right) d \zeta_{1}\right)\right. \\
\left.\quad-\sin (\alpha Y \varphi)\left(F\left(\zeta_{1}\right) d \zeta_{1}-G\left(\zeta_{2}\right) d \zeta_{2}\right)\right]=i D_{1(y)}^{\alpha} f, \\
\frac{d}{d y} \int_{0}^{x+i y}\left[\cos (\alpha Y \varphi)\left(F\left(\zeta_{1}\right) d \zeta_{1}-G\left(\zeta_{2}\right) d \zeta_{2}\right)\right. \\
\left.\quad+\sin (\alpha Y \varphi)\left(F\left(\zeta_{1}\right) d \zeta_{2}+G\left(\zeta_{2}\right) d \zeta_{1}\right)\right]=i D_{2(y)}^{\alpha} f,
\end{gathered}
$$

equation (13) is reduced to

$$
D_{(z)}^{(\alpha)} f(z)=\frac{R^{-\alpha}}{2 \Gamma(1-\alpha)}\left[D_{1(x)}^{(\alpha)} f+D_{2(y)}^{(\alpha)} f\right]+i \frac{R^{-\alpha}}{2 \Gamma(1-\alpha)}\left[D_{1(y)}^{(\alpha)} f+D_{2(x)}^{(\alpha)} f\right]
$$

$$
D_{(z)}^{(\alpha)} f(z)=\Re D_{(z)}^{(\alpha)} f(z)+i \Im D_{(z)}^{(\alpha)} f(z)=\bar{D}_{(z)}^{(\alpha)} f(z)+i \underline{D}_{(z)}^{(\alpha)} f(z)
$$

where

$$
\begin{aligned}
& \Re D_{(z)}^{(\alpha)} f(z) \equiv \bar{D}_{(z)}^{(\alpha)} f(z)=\frac{R^{-\alpha}}{2 \Gamma(1-\alpha)}\left[D_{1(x)}^{(\alpha)} f+D_{2(y)}^{(\alpha)} f\right] \\
& \Im D_{(z)}^{(\alpha)} f(z) \equiv \underline{D}_{(z)}^{(\alpha)} f(z)=\frac{R^{-\alpha}}{2 \Gamma(1-\alpha)}\left[D_{1(y)}^{(\alpha)} f+D_{2(x)}^{(\alpha)} f\right] .
\end{aligned}
$$


Lemma 2.1. The fractional complexified Euler-Lagrange equation (7) is split into two parts:

$$
\begin{aligned}
& \rho_{i}^{\eta}\left(\frac{\partial L_{1}}{\partial x_{1}^{\eta}}+\frac{\partial L_{2}}{x_{2}^{\eta}}\right) \\
& -\left(\bar{D}_{(z)}^{(\alpha)} f(z)\left(\frac{\partial L_{1}}{\partial y_{1}^{j}}+\frac{\partial L_{2}}{\partial y_{2}^{j}}\right)-\underline{D}_{(z)}^{(\alpha)} f(z)\left(\frac{\partial L_{1}}{\partial y_{1}^{j}}+\frac{\partial L_{2}}{\partial y_{2}^{j}}\right)\right) \\
& -\left(\frac{\partial L_{1}}{\partial y_{1}^{k}}+\frac{\partial L_{2}}{y_{2}^{k}}\right)\left(\bar{C}_{i j}^{k} y_{1}^{j}+\underline{C}_{i j}^{k} y_{2}^{j}\right) \\
& +\left(\frac{\partial L_{2}}{\partial y_{1}^{k}}-\frac{\partial L_{1}}{y_{2}^{k}}\right)\left(\bar{C}_{i j}^{k} y_{2}^{j}-\underline{C}_{i j}^{k} y_{1}^{j}\right) \\
& -(\alpha-1)\left[p\left(\frac{\partial L_{1}}{\partial y_{1}^{k}}+\frac{\partial L_{2}}{y_{2}^{k}}\right)-q\left(\frac{\partial L_{2}}{\partial y_{1}^{k}}-\frac{\partial L_{1}}{y_{2}^{k}}\right)\right]=0
\end{aligned}
$$

and

$$
\begin{aligned}
& \rho_{i}^{\eta}\left(\frac{\partial L_{2}}{\partial x_{1}^{\eta}}-\frac{\partial L_{1}}{x_{2}^{\eta}}\right) \\
& -\left(\bar{D}_{(z)}^{(\alpha)} f(z)\left(\frac{\partial L_{2}}{\partial y_{1}^{j}}-\frac{\partial L_{1}}{\partial y_{2}^{j}}\right)+\underline{D}_{(z)}^{(\alpha)} f(z)\left(\frac{\partial L_{1}}{\partial y_{1}^{j}}+\frac{\partial L_{2}}{\partial y_{2}^{j}}\right)\right) \\
& -\left(\frac{\partial L_{2}}{\partial y_{1}^{k}}-\frac{\partial L_{1}}{y_{2}^{k}}\right)\left(\bar{C}_{i j}^{k} y_{1}^{j}+\underline{C}_{i j}^{k} y_{2}^{j}\right) \\
& -\left(\frac{\partial L_{1}}{\partial y_{1}^{k}}+\frac{\partial L_{2}}{y_{2}^{k}}\right)\left(\bar{C}_{i j}^{k} y_{2}^{j}-\underline{C}_{i j}^{k} y_{1}^{j}\right) \\
& -(\alpha-1)\left[q\left(\frac{\partial L_{1}}{\partial y_{1}^{k}}+\frac{\partial L_{2}}{y_{2}^{k}}\right)+p\left(\frac{\partial L_{2}}{\partial y_{1}^{k}}-\frac{\partial L_{1}}{y_{2}^{k}}\right)\right]=0,
\end{aligned}
$$

where

$$
\begin{gathered}
\sinh \left(\zeta_{1}+i \zeta_{2}\right)=\sinh \zeta_{1} \cos \zeta_{2}+i \cosh \zeta_{1} \sin \zeta_{2}=A+i B, \\
p=\frac{A X+B Y}{X^{2}+Y^{2}}, \\
q=\frac{B X-A Y}{X^{2}+Y^{2}} .
\end{gathered}
$$

Remark 2.3. Making use of the previous arguments, one may already notice that the complexified action (3) may also be split into a real and a complexified part. We expect that the emergent imaginary part of the action will contribute as a corrector to the real part when applied to a physical problem and exotic solutions that will arise from the complexified counterpart may have physical relevance $[18,19]$. 
Remark 2.4. We expect that equations (18) and (19) will play a crucial role when dealing with dissipative dynamical systems on complex Lie algebroids, in particular those dynamical systems exhibiting oscillatory motion.

Remark 2.5. For mathematical convenience and clarification, we will use in the rest of the manuscript the following notations:

$$
\begin{gathered}
\frac{\bar{\partial}}{\overline{\bar{\partial}} x_{1}^{\eta}} \equiv \bar{D}^{(\alpha)}, \\
\frac{\underline{\partial}}{\underline{\partial} x_{1}^{\eta}} \equiv \underline{D}^{(\alpha)},
\end{gathered}
$$

where $\bar{D}^{(\alpha)}$ and $\underline{D}^{(\alpha)}$ are defined by equation (14).

As an exemplification, we will derive the fractional geodesics for complex Lie algebroids. In complexified coordinates, the complexified Lagrangian

$$
L=\frac{1}{2} g_{i j}(x) y^{i} y^{j}=L_{1}+i L_{2},
$$

is expected to induce an isomorphism of the complex vector (dual $E^{*}$ ) bundles $\tilde{g}: E \rightarrow E^{*}$. Here the metric

$$
g=g_{i j}(x) e^{i} \otimes e^{j},
$$

is complexified, i.e. $g=g_{1}+i g_{2}$. The fractional complexified EulerLagrange equations (15) and (16) now read:

$$
\begin{aligned}
\frac{1}{2} \frac{\bar{\partial}}{\bar{\partial} x_{1}^{\eta}}\left[g_{i k 1} y_{1}^{i}-g_{i k 2} y_{2}^{i}\right]+\frac{1}{2} \frac{\underline{\partial}}{\partial} x_{2}^{\eta}\left[g_{i k 1} y_{1}^{i}-g_{i k 2} y_{2}^{i}\right] \\
=(\alpha-1)\left[p\left(g_{i k 1} y_{1}^{i}-g_{i k 2} y_{2}^{i}-q\left(g_{i k 1} y_{2}^{i}+g_{i k 2} y_{1}^{i}\right)\right]\right. \\
+\left[\bar{C}_{i k}^{j} g_{s j 1}+\underline{C}_{i k}^{j} g_{s j 2}+\frac{1}{4} \rho_{k}^{\eta}\left(\frac{\bar{\partial} g_{i j 1}}{\bar{\partial} x_{1}^{\eta}}+\frac{\underline{\partial} g_{i j 2}}{\underline{\partial} x_{2}^{\eta}}\right)\right]\left[y_{1}^{i} y_{1}^{j}-y_{2}^{i} y_{2}^{j}\right] \\
-\left[\bar{C}_{i k}^{j} g_{s j 2}+\underline{C}_{i k}^{j} g_{s j 1}+\frac{1}{4} \rho_{k}^{\eta}\left(\frac{\bar{\partial} g_{i j 2}}{\bar{\partial} x_{1}^{\eta}}-\frac{\underline{\partial} g_{i j 1}}{\underline{\partial} x_{2}^{\eta}}\right)\right]\left[y_{1}^{i} y_{2}^{j}+y_{2}^{i} y_{1}^{j}\right]
\end{aligned}
$$

and

$$
\begin{aligned}
\frac{1}{2} \frac{\bar{\partial}}{\bar{\partial} x_{1}^{\eta}}\left[g_{i k 1} y_{2}^{i}+g_{i k 2} y_{1}^{i}\right]-\frac{1}{2} \frac{\underline{\partial}}{\partial x_{2}^{\eta}}\left[g_{i k 1} y_{1}^{i}-g_{i k 2} y_{2}^{i}\right] \\
=(\alpha-1)\left[p\left(g_{i k 1} y_{2}^{i}+g_{i k 2} y_{1}^{i}\right)+q\left(g_{i k 1} y_{1}^{i}+g_{i k 2} y_{2}^{i}\right)\right] \\
+\left[\bar{C}_{i k}^{j} g_{s j 2}-\underline{C}_{i k}^{j} g_{s j 1}+\frac{1}{4} \rho_{k}^{\eta}\left(\frac{\bar{\partial} g_{i j 2}}{\bar{\partial} x_{1}^{\eta}}-\frac{\partial}{\underline{\partial} g_{i j 1}}\right)\right]\left[y_{1}^{i} y_{1}^{j}-y_{2}^{i} y_{2}^{j}\right] \\
-\left[\bar{C}_{i k}^{j} g_{s j 1}+\underline{C}_{i k}^{j} g_{s j 2}+\frac{1}{4} \rho_{k}^{\eta}\left(\frac{\bar{\partial} g_{i j 1}}{\bar{\partial} x_{1}^{\eta}}+\frac{\underline{\partial} g_{i j 2}}{\underline{\partial} x_{2}^{\eta}}\right)\right]\left[y_{1}^{i} y_{2}^{j}+y_{2}^{i} y_{1}^{j}\right],
\end{aligned}
$$

which are the complexified geodesics equations. 
One more illustration concerns the Wong's equations which arise in the dynamics of a colored particle in Yang-Mills field and on the falling cat theorem [45]. The complexified Lagrangian and Hamiltonian of the theory on the complex Lie algebroid $E$ are given by

$$
\begin{aligned}
& L\left(x^{\eta}(z), D_{(z)}^{(\alpha)} x^{\eta}(z), \overline{\mathrm{v}}^{i}(z)\right)=\frac{1}{2} h_{i j} \overline{\mathrm{v}}^{i} \overline{\mathrm{v}}^{j}+\frac{1}{2} g_{\eta \sigma} u^{\eta} u^{\sigma} \\
& =\frac{1}{2}\left(h_{i j}^{1}\left(\overline{\mathrm{v}}_{1}^{i} \overline{\mathrm{v}}_{1}^{j}-\overline{\mathrm{v}}_{2}^{i} \overline{\mathrm{v}}_{2}^{j}\right)-h_{i j}^{2}\left(\overline{\mathrm{v}}_{1}^{i} \overline{\mathrm{v}}_{2}^{j}+\overline{\mathrm{v}}_{2}^{i} \overline{\mathrm{v}}_{1}^{j}\right)\right) \\
& \left.+i\left[h_{i j}^{1}\left(\overline{\mathrm{v}}_{1}^{i} \overline{\mathrm{v}}_{2}^{j}+\overline{\mathrm{v}}_{2}^{i} \overline{\mathrm{v}}_{1}^{j}\right)+h_{i j}^{2}\left(\overline{\mathrm{v}}_{1}^{i} \overline{\mathrm{v}}_{1}^{j}-\overline{\mathrm{v}}_{2}^{i} \overline{\mathrm{v}}_{2}^{j}\right)\right]\right) \\
& +\frac{1}{2}\left(g_{\eta \sigma}^{1}\left(u_{1}^{\eta} u_{1}^{\sigma}-u_{2}^{\eta} u_{2}^{\sigma}\right)-g_{\eta \sigma}^{2}\left(u_{1}^{\eta} u_{2}^{\sigma}+u_{2}^{\eta} u_{1}^{\sigma}\right)\right) \\
& +i\left[g_{\eta \sigma}^{1}\left(u_{1}^{\eta} u_{2}^{\sigma}+u_{2}^{\eta} u_{1}^{\sigma}\right)+g_{\eta \sigma}^{2}\left(u_{1}^{\eta} u_{1}^{\sigma}-u_{2}^{\eta} u_{2}^{\sigma}\right)\right. \\
& =\underbrace{\frac{1}{2}\left(h_{i j}^{1}\left(\overline{\mathrm{v}}_{1}^{i} \overline{\mathrm{v}}_{1}^{j}-\overline{\mathrm{v}}_{2}^{i} \overline{\mathrm{v}}_{2}^{j}\right)-h_{i j}^{2}\left(\overline{\mathrm{v}}_{1}^{i} \overline{\mathrm{v}}_{2}^{j}+\overline{\mathrm{v}}_{2}^{i} \overline{\mathrm{v}}_{1}^{j}\right)\right)}_{L_{1}(h(z))} \\
& +\underbrace{\frac{1}{2}\left(g_{\eta \sigma}^{1}\left(u_{1}^{\eta} u_{1}^{\sigma}-u_{2}^{\eta} u_{2}^{\sigma}\right)-g_{\eta \sigma}^{2}\left(u_{1}^{\eta} u_{2}^{\sigma}+u_{2}^{\eta} u_{1}^{\sigma}\right)\right)}_{L_{1}(g(z))} \\
& +\frac{1}{2} i[\underbrace{h_{i j}^{1}\left(\overline{\mathrm{v}}_{1}^{i} \overline{\mathrm{v}}_{2}^{j}+\overline{\mathrm{v}}_{2}^{i} \overline{\mathrm{v}}_{1}^{j}\right)+h_{i j}^{2}\left(\overline{\mathrm{v}}_{1}^{i} \overline{\mathrm{v}}_{1}^{j}-\overline{\mathrm{v}}_{2}^{i} \overline{\mathrm{v}}_{2}^{j}\right)}_{L_{2}(h(z))} \\
& +\underbrace{g_{\eta \sigma}^{1}\left(u_{1}^{\eta} u_{2}^{\sigma}+u_{2}^{\eta} u_{1}^{\sigma}\right)+g_{\eta \sigma}^{2}\left(u_{1}^{\eta} u_{1}^{\sigma}-u_{2}^{\eta} u_{2}^{\sigma}\right)}_{L_{2}(g(z))}] \\
& =L_{1}(h(z))+L_{1}(g(z))+i\left[L_{2}(h(z))+L_{2}(g(z))\right]
\end{aligned}
$$

for $x^{\eta}=x_{1}^{\eta}+i x_{2}^{\eta} ; \overline{\mathrm{v}}^{i}=\overline{\mathrm{v}}_{1}^{i}+i \overline{\mathrm{v}}_{2}^{i} ; u^{\eta}=u_{1}^{\eta}+i u_{2}^{\eta}$ and

$$
\begin{aligned}
\mathbf{H}( & \left.x^{\eta}(z), p_{\eta}(z), \bar{p}_{i}(z)\right)=\frac{1}{2} h^{i j} \bar{p}_{i} \bar{p}_{j}+\frac{1}{2} g^{\eta \sigma} p_{\eta} p_{\sigma}, \\
= & \underbrace{\frac{1}{2}\left(h_{1}^{i j}\left(\bar{p}_{i}^{1} \bar{p}_{j}^{1}-\bar{p}_{i}^{2} \bar{p}_{j}^{2}\right)-h_{2}^{i j}\left(\bar{p}_{i}^{1} \bar{p}_{j}^{2}+\bar{p}_{i}^{2} \bar{p}_{j}^{1}\right)\right)}_{H_{1}(h(z))} \\
& +\underbrace{\frac{1}{2}\left(g_{1}^{\eta \sigma}\left(p_{\eta}^{1} p_{\sigma}^{1}-p_{\eta}^{2} p_{\sigma}^{2}\right)-g_{2}^{\eta \sigma}\left(p_{\eta}^{1} p_{\sigma}^{2}+p_{\eta}^{2} p_{\sigma}^{1}\right)\right)}_{H_{1}(g(z))}
\end{aligned}
$$




$$
\begin{aligned}
+\frac{1}{2} i[\underbrace{h_{2}^{i j}\left(\bar{p}_{i}^{1} \bar{p}_{j}^{2}+\bar{p}_{i}^{2} \bar{p}_{j}^{1}\right)+h_{2}^{i j}\left(\bar{p}_{i}^{1} \bar{p}_{j}^{1}-\bar{p}_{i}^{2} \bar{p}_{j}^{2}\right)}_{H_{2}(h(z))} \\
+\underbrace{g_{1}^{\eta \sigma}\left(p_{\eta}^{1} p_{\sigma}^{2}+p_{\eta}^{2} p_{\sigma}^{1}\right)+g_{2}^{\eta \sigma}\left(p_{\eta}^{1} p_{\sigma}^{1}-p_{\eta}^{2} p_{\sigma}^{2}\right)}_{H_{2}(g(z))}] \\
=\mathbf{H}_{1}(h(z))+\mathbf{H}_{1}(g(z))+i\left[\mathbf{H}_{2}(h(z))+\mathbf{H}_{2}(g(z))\right]
\end{aligned}
$$

for $x^{\eta}=x_{1}^{\eta}+i x_{2}^{\eta}$ and $\bar{p}^{i}=\bar{p}_{1}^{i}+i \bar{p}_{2}^{i}$, where $u=\dot{x}$,

$$
\left(x^{\eta}, \dot{x}^{\eta}, \overline{\mathrm{v}}^{i}\right) \equiv\left(x_{1}^{\eta}+i x_{2}^{\eta}, \dot{x}_{1}^{\eta}+i \dot{x}_{2}^{\eta}, \overline{\mathrm{v}}_{1}^{i}+i \overline{\mathrm{v}}_{2}^{i}\right),
$$

is the analogous dual fibered complexified coordinates on $T Q / G$ and

$$
\left(x^{\eta}, p_{\eta}, \bar{p}_{i}\right) \equiv\left(x_{1}^{\eta}+i x_{2}^{\eta}, p_{\eta}^{1}+i p_{\eta}^{2}, \bar{p}_{i}^{1}+i \bar{p}_{i}^{2}\right),
$$

is the dual complexified coordinates on $T^{*} Q / G, G$ being a compact Lie group, $E=T M \times \mathcal{A}$ with $\operatorname{dim} T M=m$ and $\operatorname{dim} \mathcal{A}=n, \mathcal{A}$ being an arbitrary Lie $\mathbb{C}$-algebra of complex dimension $n$. $h$ is a complex metric on $\mathcal{A}$ and $g$ is assumed to be a Riemannian complex metric on $M$ with $h=h_{1}+i h_{2}$ and $g=g_{1}+i g_{2}$. We may now derive the complexified Wong's equation on the complex plane which is split into the real and complex parts correspondingly:

$$
\begin{aligned}
& \frac{\bar{d} \overline{\mathrm{v}}_{1}^{i}}{\bar{d} \zeta}=\bar{C}_{j i}^{l}\left[\left(h_{1}^{i k} h_{l s 1}-h_{2}^{i k} h_{l s 2}\right)\left(\overline{\mathrm{v}}_{1}^{j} \overline{\mathrm{v}}_{1}^{s}-\overline{\mathrm{v}}_{2}^{j} \overline{\mathrm{v}}_{2}^{s}\right)\right. \\
& \left.-\left(h_{1}^{i k} h_{l s 2}+h_{2}^{i k} h_{l s 1}\right)\left(\overline{\mathrm{v}}_{1}^{j} \overline{\mathrm{v}}_{2}^{s}+\overline{\mathrm{v}}_{2}^{j} \overline{\mathrm{v}}_{1}^{s}\right)\right] \\
& +\underline{C}_{j i}^{l}\left[\left(h_{1}^{i k} h_{l s 1}-h_{2}^{i k} h_{l s 2}\right)\left(\overline{\mathrm{v}}_{1}^{j} \overline{\mathrm{v}}_{2}^{s}+\overline{\mathrm{v}}_{2}^{j} \overline{\mathrm{v}}_{1}^{s}\right)\right. \\
& \left.+\left(h_{1}^{i k} h_{l s 2}+h_{2}^{i k} h_{l s 1}\right)\left(\overline{\mathrm{v}}_{1}^{j} \overline{\mathrm{v}}_{1}^{s}-\overline{\mathrm{v}}_{2}^{j} \overline{\mathrm{v}}_{2}^{s}\right)\right]+(\alpha-1)\left[p \overline{\mathrm{v}}_{1}^{k}-q \overline{\mathrm{v}}_{2}^{k}\right], \\
& \frac{\underline{d} \overline{\mathrm{v}}_{2}^{i}}{\underline{d \zeta}}=\bar{C}_{j i}^{l}\left[\left(h_{1}^{i k} h_{l s 1}-h_{2}^{i k} h_{l s 2}\right)\left(\overline{\mathrm{v}}_{1}^{j} \overline{\mathrm{v}}_{2}^{s}+\overline{\mathrm{v}}_{2}^{j} \overline{\mathrm{v}}_{1}^{s}\right)\right. \\
& \left.+\left(h_{1}^{i k} h_{l s 2}+h_{2}^{i k} h_{l s 1}\right)\left(\overline{\mathrm{v}}_{1}^{j} \overline{\mathrm{v}}_{1}^{s}-\overline{\mathrm{v}}_{2}^{j} \overline{\mathrm{v}}_{2}^{s}\right)\right] \\
& -\underline{C}_{j i}^{l}\left[\left(h_{1}^{i k} h_{l s 1}-h_{2}^{i k} h_{l s 2}\right)\left(\overline{\mathrm{v}}_{1}^{j} \overline{\mathrm{v}}_{1}^{s}-\overline{\mathrm{v}}_{2}^{j} \overline{\mathrm{v}}_{2}^{s}\right)\right. \\
& \left.-\left(h_{1}^{i k} h_{l s 2}+h_{2}^{i k} h_{l s 1}\right)\left(\overline{\mathrm{v}}_{1}^{j} \overline{\mathrm{v}}_{2}^{s}+\overline{\mathrm{v}}_{2}^{j} \overline{\mathrm{v}}_{1}^{s}\right)\right]+(\alpha-1)\left[p \overline{\mathrm{v}}_{1}^{k}+q \overline{\mathrm{v}}_{2}^{k}\right] .
\end{aligned}
$$

A number of applications of the complexified geodesics equations and complexified Wong's equations within the framework of quantum field theory are under advancement. 
3. Complexified hamiltonian for complex Lie algebroids. Let $L\left(\zeta, q(\zeta), D_{(z)}^{(\alpha)} q(\zeta)\right)$ be a hyperregular complexified Lagrangian on the complex Lie algebroid $E$ with admissible curve $q$ in $E$ and with two fixed endpoints

$$
A, B \in M \subset \mathcal{P}(J, E)_{A}^{B}=\{\mathcal{P}(J, E) \mid \pi(q(0))=A \text { and } \pi(q(z))=B\},
$$

and

$$
\mathcal{H}\left(\zeta, q(\zeta), D_{(z)}^{(\alpha)} q(\zeta)\right)=p_{i} y^{i}-L\left(\zeta, q(\zeta), D_{(z)}^{(\alpha)} q(\zeta)\right)
$$

where

$$
p_{i}=\frac{\partial\left(\zeta, q(\zeta), D_{(z)}^{(\alpha)} q(\zeta)\right)}{\partial y^{i}},
$$

be the corresponding complexified Hamiltonian. If $(q, \dot{q})$ is the corresponding minimizer, then there exists $p$ such that the generalized complexified Hamiltonian system for complex Lie algebroids:

$$
\begin{aligned}
\frac{d x^{\alpha}(\zeta)}{d \zeta}= & \rho_{i}^{\eta} \frac{\partial \mathcal{H}}{\partial p_{i}}\left(\zeta, q(\zeta), D_{(z)}^{(\alpha)} q(\zeta)\right) \\
\frac{d p_{i}(\zeta)}{d \zeta}= & -\rho_{i}^{\eta} \frac{\partial \mathcal{H}}{\partial x^{\eta}}\left(\zeta, q(\zeta), D_{(z)}^{(\alpha)} q(\zeta)\right) \\
& -\frac{(\alpha-1) \sinh \zeta}{\cosh z-\cosh \zeta} p_{i}-C_{i, j}^{k} p_{k}(\zeta) \frac{\partial \mathcal{H}}{\partial p_{j}}\left(\zeta, q(\zeta), D_{(z)}^{(\alpha)} q(\zeta)\right)
\end{aligned}
$$

Their complexified parts are accordingly:

$$
\begin{gathered}
\frac{\bar{d} x_{1}^{\alpha}}{\bar{d} \zeta_{1}}+\frac{\underline{d} x_{2}^{\alpha}}{\underline{d} \zeta_{2}}=2 \rho_{i}^{\eta}\left[\frac{\bar{\partial} \mathcal{H}_{1}}{\bar{\partial} p_{j}^{1}}+\frac{\underline{\partial \mathcal{H}_{2}}}{\underline{\partial} p_{j}^{2}}\right], \\
\frac{\bar{d} x_{2}^{\alpha}}{\bar{d} \zeta_{1}}-\frac{\underline{d} x_{1}^{\alpha}}{\underline{d} \zeta_{2}}=2 \rho_{i}^{\eta}\left[\frac{\bar{\partial} \mathcal{H}_{2}}{\bar{\partial} p_{j}^{1}}-\frac{\partial \mathcal{H}_{1}}{\underline{\partial} p_{j}^{2}}\right], \\
\frac{\bar{d} p_{i, 1}}{\bar{d} \zeta_{1}}+\frac{d p_{i, 2}}{\underline{d} \zeta_{2}}=-\frac{1}{2} \rho_{i}^{\eta} \frac{\partial \mathcal{H}_{1}}{\partial x_{1}^{\eta}}-(\alpha-1)\left(p p_{i, 1}-q p_{i, 2}\right) \\
-\frac{1}{2}\left[\bar{C}_{i, j}^{k}\left(p_{k, 1} \frac{\partial \mathcal{H}_{1}}{\partial p_{j, 1}}+p_{k, 2} \frac{\partial \mathcal{H}_{2}}{\partial p_{j, 2}}\right)+\underline{C}_{i, j}^{k}\left(p_{k, 2} \frac{\partial \mathcal{H}_{1}}{\partial p_{j, 1}}-p_{k, 1} \frac{\partial \mathcal{H}_{2}}{\partial p_{j, 2}}\right)\right], \\
\frac{\bar{d} p_{i, 1}}{\bar{d} \zeta_{1}}-\frac{d p_{i, 1}}{\underline{d} \zeta_{2}}=\frac{1}{2} \rho_{i}^{\eta} \frac{\partial \mathcal{H}_{2}}{\partial x_{2}^{\eta}}-(\alpha-1)\left(p p_{i, 2}+q p_{i, 1}\right) \\
-\frac{1}{2}\left[\bar{C}_{i, j}^{k}\left(p_{k, 2} \frac{\partial \mathcal{H}_{1}}{\partial p_{j, 1}}-p_{k, 1} \frac{\partial \mathcal{H}_{2}}{\partial p_{j, 2}}\right)-\underline{C}_{i, j}^{k}\left(p_{k, 1} \frac{\partial \mathcal{H}_{1}}{\partial p_{j, 1}}+p_{k, 2} \frac{\partial \mathcal{H}_{2}}{\partial p_{j, 2}}\right)\right] .
\end{gathered}
$$


Lemma 3.1. The fractional complexified Hamiltonian dynamics on the complex dual bundle $E^{*}$ is represented by the complex vector fields:

$$
\begin{aligned}
& \mathcal{D}^{r}(z, \zeta)=\rho_{i}^{\eta} \frac{\partial \mathcal{H}}{\partial p_{i}} \frac{\partial}{\partial x^{\eta}}-(\alpha-1)(p+i q) \frac{\partial}{\partial \zeta_{i}}-\left(\rho_{i}^{\eta} \frac{\partial \mathcal{H}}{\partial x^{\eta}}+C_{i, j}^{k} p_{k}(t) \frac{\partial \mathcal{H}}{\partial p_{j}}\right) \frac{\partial}{\partial \zeta_{i}} \\
& =\frac{1}{2} \rho_{i}^{\eta}\left[\left(\frac{\bar{\partial} \mathcal{H}_{1}}{\bar{\partial} p_{j}^{1}}+\frac{\underline{\partial} \mathcal{H}_{2}}{\partial p_{j}^{2}}\right) \frac{\partial}{\partial x_{1}^{\eta}}+\left(\frac{\bar{\partial} \mathcal{H}_{2}}{\bar{\partial} p_{j}^{1}}-\frac{\partial \mathcal{H}_{1}}{\partial p_{j}^{2}}\right) \frac{\partial}{\partial x_{2}^{\eta}}\right] \\
& -\frac{1}{2}(\alpha-1)\left[p \frac{\partial}{\partial \zeta_{i, 1}}+q \frac{\partial}{\partial \zeta_{i, 2}}\right] \\
& -\frac{1}{2} \rho_{i}^{\eta}\left(\frac{\bar{\partial} \mathcal{H}_{1}}{\bar{\partial} x_{1}^{\eta}}+\frac{\partial \mathcal{H}_{2}}{\underline{\partial} x_{2}^{\eta}}\right) \frac{\partial}{\partial \zeta_{1}}+\frac{1}{2} \rho_{i}^{\eta}\left(\frac{\bar{\partial} \mathcal{H}_{2}}{\bar{\partial} x_{1}^{\eta}}-\frac{\partial \mathcal{H}_{1}}{\underline{\partial} x_{2}^{\eta}}\right) \frac{\partial}{\partial \zeta_{2}} \\
& -\frac{1}{2}\left(\bar{C}_{i, j}^{k} p_{k, 1}+\underline{C}_{i, j}^{k} p_{k, 2}\right)\left[\left(\frac{\bar{\partial} \mathcal{H}_{1}}{\bar{\partial} p_{j}^{1}}+\frac{\underline{\partial \mathcal{H}_{2}}}{\underline{\partial} p_{j}^{2}}\right) \frac{\partial}{\partial \zeta_{1}}+\left(\frac{\bar{\partial} \mathcal{H}_{2}}{\bar{\partial} p_{j}^{1}}-\frac{\underline{\partial \mathcal{H}_{1}}}{\underline{\partial} p_{j}^{2}}\right) \frac{\partial}{\partial \zeta_{2}}\right] \\
& +\frac{1}{2}\left(\bar{C}_{i, j}^{k} p_{k, 2}-\underline{C}_{i, j}^{k} p_{k, 1}\right)\left[\left(\frac{\bar{\partial} \mathcal{H}_{2}}{\bar{\partial} p_{j}^{1}}-\frac{\partial \mathcal{H}_{1}}{\underline{\partial} p_{j}^{2}}\right) \frac{\partial}{\partial \zeta_{1}}-\left(\frac{\bar{\partial} \mathcal{H}_{1}}{\bar{\partial} p_{j}^{1}}+\frac{\underline{\partial} \mathcal{H}_{2}}{\underline{\partial} p_{j}^{2}}\right) \frac{\partial}{\partial \zeta_{2}}\right] \\
& +i\left\{\frac{1}{2} \rho_{i}^{\eta}\left[\left(\frac{\bar{\partial} \mathcal{H}_{2}}{\bar{\partial} p_{j}^{1}}-\frac{\partial \mathcal{H}_{1}}{\underline{\partial} p_{j}^{2}}\right) \frac{\partial}{\partial x_{1}^{\eta}}-\left(\frac{\bar{\partial} \mathcal{H}_{1}}{\bar{\partial} p_{j}^{1}}+\frac{\partial \mathcal{H}_{2}}{\partial p_{j}^{2}}\right) \frac{\partial}{\partial x_{2}^{\eta}}\right]\right. \\
& -\frac{1}{2}(\alpha-1)\left(q \frac{\partial}{\partial \zeta_{i, 1}}-p \frac{\partial}{\partial \zeta_{i, 2}}\right) \\
& -\frac{1}{2} \rho_{i}^{\eta}\left(\frac{\bar{\partial} \mathcal{H}_{2}}{\bar{\partial} x_{1}^{\eta}}-\frac{\partial \mathcal{H}_{1}}{\underline{\partial} x_{2}^{\eta}}\right) \frac{\partial}{\partial \zeta_{1}}+\frac{1}{2} \rho_{i}^{\eta}\left(\frac{\bar{\partial} \mathcal{H}_{1}}{\bar{\partial} x_{1}^{\eta}}+\frac{\partial \mathcal{H}_{2}}{\underline{\partial} x_{2}^{\eta}}\right) \frac{\partial}{\partial \zeta_{2}} \\
& -\frac{1}{2}\left(\bar{C}_{i, j}^{k} p_{k, 1}+\underline{C}_{i, j}^{k} p_{k, 2}\right)\left[\left(\frac{\bar{\partial} \mathcal{H}_{2}}{\bar{\partial} p_{j}^{1}}-\frac{\underline{\partial \mathcal{H}_{1}}}{\underline{\partial} p_{j}^{2}}\right) \frac{\partial}{\partial \zeta_{1}}-\left(\frac{\bar{\partial} \mathcal{H}_{1}}{\bar{\partial} p_{j}^{1}}+\frac{\underline{\partial \mathcal{H}_{2}}}{\underline{\partial} p_{j}^{2}}\right) \frac{\partial}{\partial \zeta_{2}}\right] \\
& \left.-\frac{1}{2}\left(\bar{C}_{i, j}^{k} p_{k, 1}-\underline{C}_{i, j}^{k} p_{k, 2}\right)\left[\left(\frac{\bar{\partial} \mathcal{H}_{1}}{\bar{\partial} p_{j}^{1}}+\frac{\partial \mathcal{H}_{2}}{\underline{\partial} p_{j}^{2}}\right) \frac{\partial}{\partial \zeta_{1}}+\left(\frac{\bar{\partial} \mathcal{H}_{2}}{\bar{\partial} p_{j}^{1}}-\frac{\underline{\partial \mathcal{H}_{1}}}{\underline{\partial} p_{j}^{2}}\right) \frac{\partial}{\partial \zeta_{2}}\right]\right\} \\
& =\mathfrak{R} \mathcal{D}^{r}(z, \zeta)+i \mathfrak{T} \mathcal{D}^{r}(z, \zeta) \equiv \overline{\mathcal{D}}^{r}(z, \zeta)+i \underline{\mathcal{D}}^{r}(z, \zeta) \text {. }
\end{aligned}
$$

4. Conclusions and perspectives. In our opinion, this work represents the first attempt to construct the theoretical framework of complex Lie algebroids based on extended fractional variational formalism. That will be the foundation, we believe however, of more stimulating investigations. The complexified fractional Euler-Lagrange equations, the complexified Hamilton equations and the complexified geodesics and Wong's equations for complex Lie algebroids are derived. It is the author's speculations that they could have motivating outcomes on both geometric dynamics and quantum field theory $[11,13,14,15,22]$. The new class of complexified Lagrangian and Hamiltonian systems obtained on complex Lie algebroids is wider than 
the standard class of Lagrangian and Hamiltonian dynamical systems. Decomplexification of the dynamical equations, in particular the fractional Euler-Lagrange equations, geodesic equations and Wong's equations could be realized if the action is complexified in the theory. The occurrence of the new complexified action in the theory could have considerable consequences in quantum field theory, where the complex part of the fractional action may guide to a novel complexified dynamics which may well differ entirely from the classical mechanics cardinally. From a phenomenological standpoint, we can go further and construct a fractional complexified Hamiltonian approach to holomorphic Poisson manifolds and holomorphic complex Lie algebroids from the viewpoint of real Poisson geometry. We expect they will open up in the future a new inspiring research area in diverse branches of physics and supply us with a powerful tool to figure out many basic problems in the area of complexified dynamical systems and noncommutative geometry. Future research efforts may be directed towards formulating predictions that can be tracked and tested numerically. The results in this work declare additional oversimplification and advocate a supplementary study.

\section{REFERENCES}

[1] Agrawal, O. P., Fractional variational calculus and the transversality conditions, J. Phys. A 39 (2006), 10375-10384.

[2] Agrawal, O. P., Fractional variational calculus in terms of Riesz fractional derivatives, J. Phys. A 40 (2007), 6287-6303.

[3] Agrawal, O. P., Tenreiro Machado, J. A. and Sabatier, J. (Editors), Fractional Derivatives and their Applications, Nonlinear Dynamics 38, no. 1-4 (2004).

[4] Almeida, R., Malinowska, A. B. and Torres, D. F. M., A fractional calculus of variations for multiple integrals with application to vibrating string, J. Math. Phys. 51, no. 3, 033503 (2010), 12 pp.

[5] Atanacković, T. M., Konjik, S. and Pilipović, S., Variational problems with fractional derivatives: Euler-Lagrange equations, J. Phys. A 41, no. 9, 095201 (2008), 12 pp.

[6] Baleanu, D., Agrawal, O. P., Fractional Hamilton formalism within Caputo's derivative, Czechoslovak J. Phys. 56, no. 10-11 (2006), 1087-1092.

[7] Cannas da Silva, A., Weinstein, A., Geometric models for noncommutative algebras, Berkeley Mathematics Lecture Notes, 10. American Mathematical Society, Providence, 1999.

[8] Connes, A., A survey of foliations and operator algebras. Operator algebras and applications, Part I, pp. 521-628, Proc. Sympos. Pure Math. 38, Amer. Math. Soc., Providence, R. I., (1982).

[9] El-Nabulsi, R. A., A fractional approach to nonconservative Lagrangian dynamical systems, Fizika A 14, no. 4, (2005) 289-298.

[10] El-Nabulsi, R. A., A fractional action-like variational approach of some classical, quantum and geometrical dynamics, Int. J. Appl. Math. 17 (2005), 299-317.

[11] El-Nabulsi, R. A., Fractional field theories from multi-dimensional fractional variational problems, Int. J. Geom. Methods Mod. Phys. 5 (2008), 863-892.

[12] El-Nabulsi, R. A., Complexified dynamical systems from real fractional actionlike with time-dependent fractional dimension on multifractal sets, Invited contribution to The 
3rd International Conference on Complex Systems and Applications, University of Le Havre, Le Havre, Normandy, France, June 29-July 02, (2009).

[13] El-Nabulsi, R. A., Fractional variational problems from extended exponentially fractional integral, Appl. Math. Comp. 217, no. 22 (2011), 9492-9496.

[14] El-Nabulsi, R. A., Fractional action-like variational problems in holonomic, nonholonomic and semi-holonomic constrained and dissipative dynamical systems, Chaos Solitons Fractals 42 (2009), 52-61.

[15] El-Nabulsi, R. A., Fractional action-like variational problems in holonomic, nonholonomic and semi-holonomic constrained and dissipative dynamical systems, Chaos Solitons Fractals 42 (2009), 52-61.

[16] El-Nabulsi, R. A., The fractional calculus of variations from extended Erdélyi-Kober operator, Int. J. Mod. Phys. B 23 (2009), 3349-3361.

[17] El-Nabulsi, R. A., Fractional quantum Euler-Cauchy equation in the Schrödinger picture, complexified harmonic oscillators and emergence of complexified Lagrangian and Hamiltonian dynamics, Mod. Phys. Lett. B 23 (2009), 3369-3386.

[18] El-Nabulsi, R. A., Complexified fractional heat kernel and physics beyond the spectral triplet action in noncommutative geometry, Int. J. Geom. Methods Mod. Phys. 6 (2009), 941-963.

[19] El-Nabulsi, R. A., Fractional Dirac operators and left-right fractional ChamseddineConnes spectral bosonic action principle in noncommutative geometry, Int. J. Geom. Methods Mod. Phys. 7 (2010), 95-134.

[20] El-Nabulsi, R. A., Modifications at large distances from fractional and fractal arguments, Fractals 18 (2010), 185-190.

[21] El-Nabulsi, R. A., Oscillating flat FRW dark energy dominated cosmology from periodic functional approach, Comm. Theor. Phys. 54, no. 01 (2010), 16-20.

[22] El-Nabulsi, R. A., A periodic functional approach to the calculus of variations and the problem of time-dependent damped harmonic oscillators, Appl. Math. Lett. 24, no. 10 (2011), 1647-1653.

[23] El-Nabulsi, R. A., Universal fractional Euler-Lagrange equation from a generalized fractional derivate operator, Central Europan J. Phys. 9, no. 1 (2011), 260-256.

[24] El-Nabulsi, R. A., Torres, D. F. M., Necessary optimality conditions for fractional action-like integrals of variational calculus with Riemann-Liouville derivatives of or$\operatorname{der}(\alpha, \beta)$, Math. Methods Appl. Sci. 30 (2007), 1931-1939.

[25] El-Nabulsi, R. A, Torres, D. F. M., Fractional actionlike variational problems, J. Math. Phys. 49, no. 5, 053521 (2008), 7 pp.

[26] Frasin, B. A., Some applications of fractional calculus operators to the analytic part of harmonic univalent functions, Hacet. J. Math. Stat. 34 (2005), 1-7.

[27] Frederico, G. S. F., Torres, D. F. M., Constants of motion for fractional action-like variational problems, Int. J. Appl. Math. 19 (2006), 97-104.

[28] Frederico, G. S. F., Torres, D. F. M., Non-conservative Noether's theorem for fractional action-like variational problems with intrinsic and observer times, Int. J. Ecol. Econ. Stat. 9, no. F07 (2007), 74-82.

[29] Frederico, G. S. F., Torres, D. F. M., Conservation laws for invariant functionals containing compositions, Appl. Anal. 86 (2007), 1117-1126.

[30] Frederico, G. S. F., Torres, D. F. M., A formulation of Noether's theorem for fractional problems of the calculus of variations, J. Math. Anal. Appl. 334 (2007), 834846.

[31] Frederico, G. S. F., Torres, D. F. M., Fractional optimal control in the sense of Caputo and the fractional Noether's theorem, Int. Math. Forum 3, no. 9-12 (2008), 479-493.

[32] Frederico, G. S. F., Torres, D. F. M., Fractional optimal control in the sense of Caputo and the fractional Noether's theorem, Int. Math. Forum 3, no. 9-12 (2008), 479-493. 
[33] Glöckner, H., Lie group structures on quotient groups and universal complexifications for infinite-dimensional Lie groups, J. Funct. Anal. 194 (2002), 347-409.

[34] Glöckner, H., Neeb, K. H., Banach-Lie quotients, enlargibility, and universal complexifications, J. Reine Angew. Math. 560 (2003), 1-28.

[35] Goldfain, E., Fractional dynamics, Cantorian space-time and the gauge hierarchy problem, Chaos Solitons Fractals 22 (2004), 513-520.

[36] Goldfain, E., Complexity in quantum field theory and physics beyond the standard model, Chaos Solitons Fractals 28 (2006), 913-922.

[37] Goldfain, E., Fractional dynamics and the standard model for particle physics, Commun. Nonlinear Sci. Numer. Simul. 13 (2008), 1397-1404.

[38] Gualtieri, M., Generalized complex geometry, arXiv:math/0703298v2.

[39] Gukov, S., Witten, E., Gauge theory, ramification, and the geometric Langlands program, Current developments in mathematics, 2006, 35-180, Int. Press, Somerville, MA, 2008.

[40] Herrmann, R., Fractional dynamic symmetries and the ground state properties of nuclei, arXiv:0806.2300v2.

[41] Herrmann, R., Fractional spin - a property of particles described with a fractional Schrödinger equation, arXiv:0805.3434v1.

[42] Hilfer, R. (Editor), Applications of Fractional Calculus in Physics, Word Scientific Publishing Co., River Edge, NJ, 2000.

[43] Ivan, G., Ivan, M. and Opris, D., Fractional Euler-Lagrange and fractional Wong equations for Lie algebroids, Proceedings of the 4th International Colloquium Mathematics and Numerical Physics, October 6-8, 2006, Bucharest, Romania, 73-80.

[44] Kapustin, A., Witten, E., Electric-magnetic duality and the geometric Langlands program, Commun. Number Theory Phys. 1 (2007), 1-236.

[45] Kosyakov, B. P., The field of an arbitrarily moving colored charge, Teoret. Mat. Fiz. 87 (1991), 422-425 (Russian); translation in Theoret. and Math. Phys. 87 (1991), 632-635.

[46] Landsman, L. P., Lie groupoids and Lie algebroids in physics and noncommutative geometry, J. Geom. Phys. 56 (2006), 24-54.

[47] Lübke, M., Okonek, C., Moduli spaces of simple bundles and Hermitian-Einstein connections, Math. Ann. 276 (1987), 663-674.

[48] Lübke, M., Teleman, A., The Kobayashi-Hitchin Correspondence, World Scientific Publishing Co., Inc., River Edge, NJ, 1995.

[49] Mackenzie, K. C. H., General theory of Lie groupoids and Lie algebroids, London Mathematical Society Lecture Note Series, Vol. 213, Cambridge University Press, Cambridge, 2005.

[50] Martinez, E., Lagrangian mechanics on Lie algebroids, Acta Appl. Math. 67 (2001), 295-320.

[51] Martinez, E., Geometric formulation of mechanics on Lie algebroids, Proceedings of the VIII Fall Workshop on Geometry and Physics, Medina del Campo, 1999, Publicaciones de la RSME 2, (2001) 209-222.

[52] Martinez, E., Lie algebroids in classical mechanics and optimal control, SIGMA Symmetry Integrability Geom. Methods Appl. 3 (2007), Paper 050, 17 pp.

[53] Miller, K. S., Ross, B., An Introduction to the Fractional Calculus and Fractional Differential Equations, John Wiley \& Sons Inc., New York, 1993.

[54] Oldham, K. B., Spanier, J., The Fractional Calculus. Theory and Applications of Differentiation and Integration to Arbitrary Order, Acad. Press, New York-London, 1974.

[55] Ortigueira, M. D., Tenreiro Machado, J. A. (Editors), Fractional Signal Processing and Applications, Signal Processing 83, no. 11 (2003). 
[56] Ortigueira, M. D., Tenreiro Machado, J. A. (Editors), Fractional Calculus Applications in Signals and Systems, Signal Processing 86, no. 10 (2006).

[57] Podlubny, I., Fractional Differential Equations. An Introduction to Fractional Derivatives, Fractional Differential Equations, to Methods of their Solution and Some of their Applications, Academic Press Inc., San Diego, CA, 1999.

[58] Riewe, F., Nonconservative Lagrangian and Hamiltonian mechanics, Phys. Rev. E (3) 53 (1996), 1890-1899.

[59] Samko, S., Kilbas A. and Marichev, O., Fractional Integrals and Derivatives. Theory and Applications, Gordon and Breach Science Publishers, Yverdon, 1993.

[60] Tenreiro Machado, J. A. (Editor), Fractional Order Calculus and its Applications, Nonlinear Dynamics 29, no. 1-4 (2002).

[61] Tenreiro Machado, J. A., Barbosa, R. S. (Editors), Fractional Differentiation and its Applications, J. Vib. Control 14, no. 9-10 (2008).

[62] Tenreiro Machado, J. A., Luo, A. (Editors), Discontinuous and Fractional Dynamical Systems, ASME Journal of Computational and Nonlinear Dynamics 3, no. 2 (2008).

[63] Weinstein, A., Lagrangian mechanics and groupoids, Mechanics day (Waterloo, ON, 1992), 207-231, Fields Inst. Commun., 7, Amer. Math. Soc., Providence, RI, 1996.

[64] Weinstein, A., Poisson geometry. Symplectic geometry, Differential Geom. Appl. 9, no. 1-2 (1998), 213-238.

[65] Zavada, P., Operator of fractional derivative in the complex plane, Comm. Math. Phys. 192 (1998), 261-285.

El-Nabulsi Ahmad Rami

Department of Nuclear Engineering

Cheju National University

Ara-dong 1

Jeju 690-756

South Korea

College of Mathematics and Information Science Neijiang Normal University

Neijiang

Sichuan 641112

China

e-mail: nabulsiahmadrami@yahoo.fr

Received March 31, 2010
Key Laboratory of Numerical Simulation of Sichuan Province

Neijiang

Sichuan 641112

China 